\title{
Sphenoid mucocoele - an unusual cause for headaches in a teenage boy
}

\author{
S E Adam, MB ChB; M Merven, FCORL \\ Department of Otorhinolaryngology, Faculty of Medicine and Health Sciences, Stellenbosch University, Cape Town, South Africa
}

Corresponding author: S E Adam (shaunadam1982@gmail.com)

\begin{abstract}
Isolated sphenoid sinus disease in childhood is uncommon and sphenoid mucocoeles (histologically benign, epithelium-lined, mucuscontaining sacs) are rare. They are thought to arise as a result of obstruction of the sinus ostium due to previous surgery, inflammation, trauma or irradiation, but may be idiopathic. We present a case of a sphenoid sinus mucocoele in a young boy and highlight the fact that headache may be the only symptom, therefore diagnosis may require a combination of imaging and exploratory surgery.
\end{abstract}

S Afr J Child Health 2018;12(2):76-78. DOI:10.7196/SAJCH.2018.v12i2.1450

\section{Case report}

A 15-year-old boy presented with retroorbital headaches for 5 years, right peri-orbital swelling for 3 years and diplopia for several months. He had no symptoms of chronic sinusitis, allergic rhinitis or epistaxis. He had no history of cranial trauma or surgery and reported normal sensation of smell and vision. He presented to our otorhinolaryngology clinic in 2013, but was lost to follow-up until 2016.

On examination at his presentation in 2016, he was slight of build (like his mother) weighing $29.5 \mathrm{~kg}$, which is below the $3 \mathrm{rd}$ centile for weight-for-age. He had right proptosis with infratemporal soft subdermal swelling (Figs 1A and $1 \mathrm{~B}$ ), normal visual acuity and no relative afferent pupillary defect. He experienced diplopia upon right lateral gaze, with reduced abduction and supraduction. There was reduced sensation in the distribution of the opthalmic (V1) and maxillary (V2) branches of the trigeminal nerve. On nasal endoscopy, a pale, soft, submucosal mass was visible in the nasal cavity.

Computed tomography (CT) and magnetic resonance imaging (MRI) revealed a sphenoid-based locally expansile cystic lesion (Figs 2 and 3). Extensive bony erosion and remodeling was noted. Extension intra-cranially with compression of the dura and cavernous sinus without invasion was noted. The lesion involved the orbital apex and extended into the pterygopalatine fossa and then through the pterygomaxillary fissure into the infra-temporal space where it was noted subdermally at the level of the zygoma.

A transcutaneous aspiration of this lateral facial swelling was performed in the clinic and revealed 'motor oil-like' fluid (Figs 1C and 1D). Serology for HIV and hydatid disease were negative.

A presumptive diagnosis of a mucocoele was made and an endoscopic transnasal

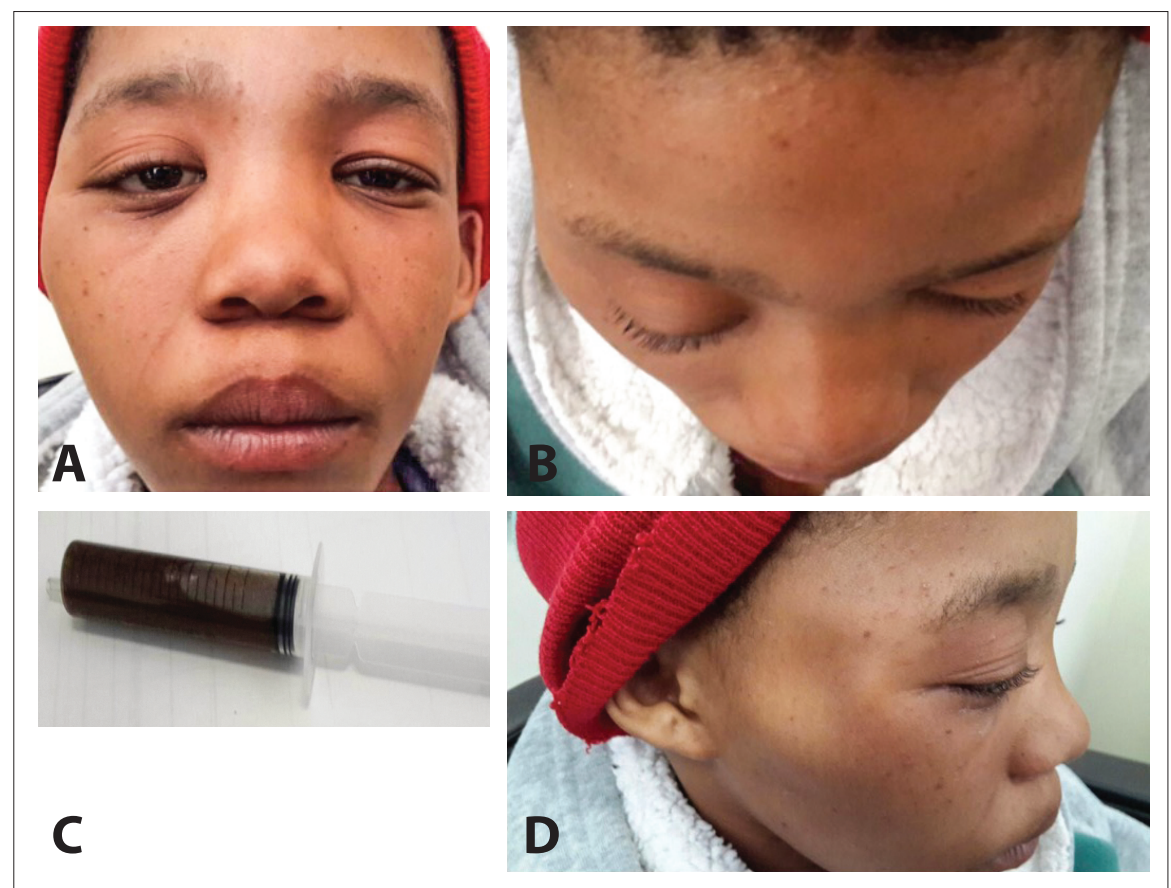

Fig. 1. (A) Frontal pre-operative view showing the right infratemporal swelling. (B) Superior view. Note right proptosis. (C) Transcutaneous aspirate fluid. (D) Post aspiration lateral view. Note the skin dimple over the area of aspiration. Informed consent was obtained to use the images.

sphenoidotomy confirmed the diagnosis. Good resolution of symptoms occurred by 1 month postoperatively. No sign of recurrence was noted clinically or on repeat $\mathrm{CT}$ at 8 months postoperatively (Fig. 4).

\section{Discussion}

Sphenoid sinuses are small recesses in the postero-superior nasal cavity and remain intra-nasal until 6 - 7 years of age. Secondary pneumatisation occurs and a true cavity is formed by 8 - 10 years of age, with maturation by $12-14$ years of age..$^{[1]}$

Paranasal sinus mucocoeles are very rare in children and sphenoid mucocoeles make up only $1-2 \%$ of all paranasal sinus mucocoeles in all age groups. ${ }^{[2]}$ To date, only 13 cases of paediatric sphenoid mucocoeles have been documented from 9 articles in the English literature.

Mucocoeles are histologically benign, epithelium-lined, mucus-containing sacs that affect the paranasal sinuses. They are thought to arise from obstruction of the sinus ostium due to previous surgery, inflammation, trauma or irradiation. Other potential aetiologies include cystic dilatation of glandular structures and cystic development from embryonic epithelial residues. ${ }^{[2]}$ Increased levels of fibroblast 

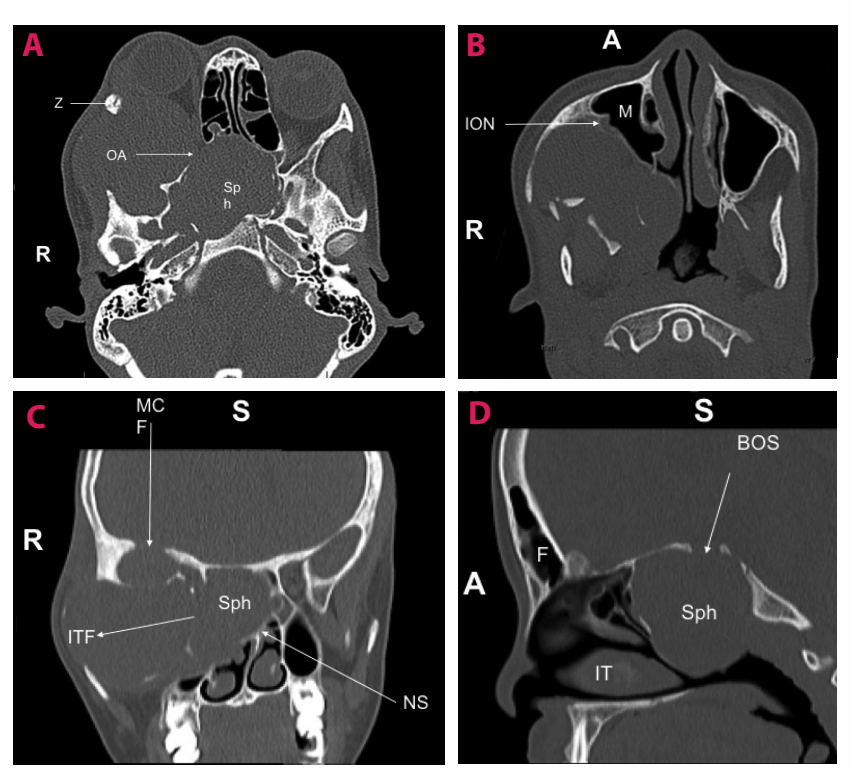

Fig. 2. (A) Axial computed tomography (CT) of orbital apex. (B) Axial CT of infra-orbital nerve (ION) relative to the right maxillary sinus (M). Note the compression of the ION by the mucocoele. (C) Coronal CT indicating the lateral extension (arrow) from the sphenoid sinus (sph) to the infra-temporal fossa (ITF) on the right. Note the deviation of the nasal septum (NS) to the left and the dehiscent middle cranial fossa (MCF) floor. (D) Sagittal CT showing the superior extent through dehiscent base of skull (BOS). ( $A=$ anterior; $S=$ superior $; O A=$ orbital apex; $Z=$ zygoma $; \quad R=$ right; $I T=$ inferior turbinate; $F=$ frontal sinus.)
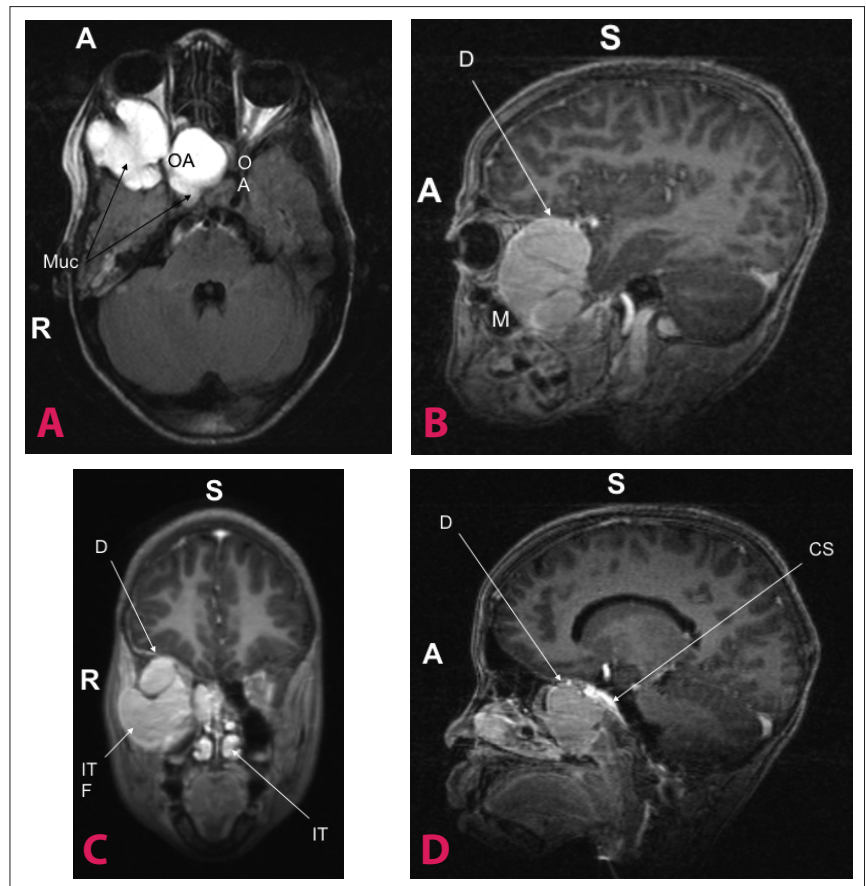

Fig. 3. (A) Axial MRI of orbital apex (OA). Note the multi-loculated mucocoele (Muc). (B) Sagittal MRI showing superior compression of the dura (D). (C) Coronal MRI showing the superior compression of dura (D) and lateral extension into infratemporal fossa (ITF). (D) Sagittal MRI indicating compression of the dura (D) superiorly and the cavernous sinus (CS) superoposteriorly. $(A=$ anterior $R=$ right $S=$ superior; $M=$ maxillary sinus; $I T=$ inferior turbinate. $)$ prostaglandin E2 and collagenase enable osteolysis by mucocoeles, facilitating their local extension. ${ }^{[3]}$

Presenting signs and symptoms are subtle until late in the disease process. In the literature, the most frequently reported manifestations are headache (89\%), decreased visual acuity (57\%), oculomotor palsies $(56 \%)$ and exophthalmos $(25 \%) .^{[4,5]}$

In this case, the dysfunction of the ophthalmic (V1) and maxillary (V2) divisions of the fifth cranial nerve (trigeminal) is unusual and would make invasive lesions more likely. However, these cranial nerve dysfunctions due to a sphenoid mucocoele have previously been reported by Yong et al. ${ }^{[6]} \mathrm{V} 1$ may be compressed or stretched at the cavernous sinus or the orbital apex where it passes through the superior orbital fissure to enter the orbit. Similarly, V2 may be involved at the cavernous sinus, pterygopalatine fossa (which houses the pterygopalatine ganglion), the pterygomaxillary fissure en route to the infratemporal fossa, or in the roof of the maxillary sinus (infra-orbital nerve). This presentation of a mucocoele with isolated lateral facial swelling due to the soft tissue extension through the abovementioned spaces is the first such report to the best of the authors' knowledge.

Mucocoeles may have variable densities on CT and signal intensities on MRI depending on their protein content, inspissation and presence of infection. MRI is best to delineate extent and internal characteristics of the lesion, whereas CT demonstrates bony involvement better. ${ }^{[7]}$ The sinus walls may be thinned or completely dehiscent with extensive advancement into surrounding structures as in this case.

Although isolated sphenoid sinus disease in childhood is rare, the differential is broad and includes inflammatory conditions (e.g. sphenoiditis, polyposis, fungal sphenoiditis, hydatid cyst); congenital conditions (e.g. dermoid cyst, epidermoid cyst, meningocoele, meningoencephalocoele, Rathkes cleft cyst, craniopharyngioma); benign lesions (e.g. mucocoele, nasopharyngeal angiofibroma, osteoma, lymphangioma, haemangioma, pituitary tumours, internal carotid artery aneurysm, fibrodysplasia) and malignant sphenoid lesions (although they have not yet been reported in this age group, but considerations would be lymphoma, rhabdomyosarcoma, Ewings sarcoma).

Differentiating a mucocoele from these may be difficult with CT and MRI, as overlap in imaging characteristics is common. In this case, a meningocoele and meningomyelocoele were excluded after imaging showed no communication with intracranial spaces. The differential included a hydatid cyst (which was excluded on serology) and lymphangioma which could not be excluded. It may only be after surgery that a definitive diagnosis can be made. An external surgical approach was used in the past but an endoscopic transnasal route, with decompression of disease, has recently become the standard of care. This approach has a lower rate of complications and is effective regardless of size and pre-operative complications. ${ }^{[8]}$

\section{Conclusion}

Childhood sphenoid mucocoele is rare and the presenting symptoms may be vague. A headache associated with other neurological symptoms or signs warrants specialist review with endoscopy and/ or further imaging. Combination CT and MRI is appropriate, but is not diagnostic in all cases. Even extensive or complicated disease is managed both safely and effectively with a diligent endoscopic transnasal approach.

\section{Learning points}

Isolated sphenoid sinus disease is very rare in children.

- A deep-seated, retro-orbital headache in a child is suggestive of sphenoid sinus disease and warrants further investigation, especially if associated with other neurological symptoms or signs. 


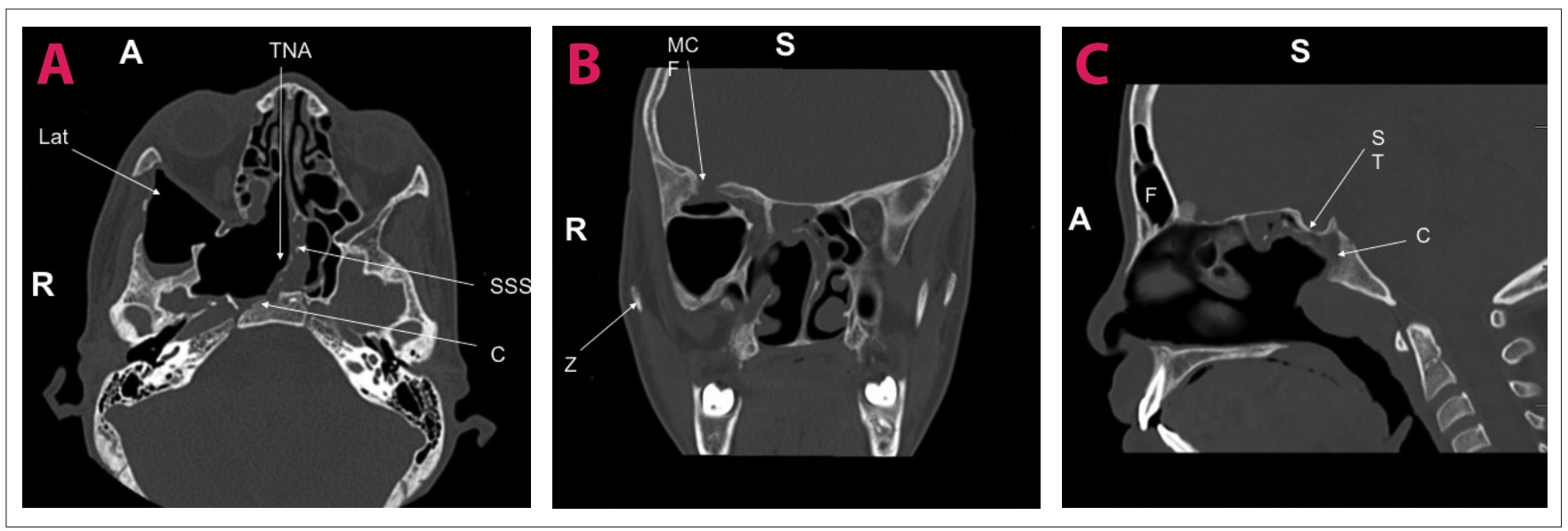

Fig. 4. Postoperative axial computed tomography (CT) indicating the lateral extent of the mucocoele (Lat), which is now aerated space. The sphenoid sinus septum (SSS) is pushed across to the left side and the eroded clivus (C). (B) Postoperative coronal CT with dehiscent middle cranial fossa (MCF) floor and zygoma (Z). (C) Postoperative sagittal CT. Note the clivus $(\mathrm{Cl})$ erosion. (TNA = transnasal approach (surgical route of access); $A=$ anterior; $R=$ right; $F=$ frontal sinus; $S T=$ sella turcica; $S=$ superior.)

- Cranial neuropathies must be excluded: optic > abducens > trigeminal $>$ oculomotor $>$ trochlear, ${ }^{[9]}$ as these may be indicative of invasive disease, and would warrant urgent referral.

- Imaging is helpful but may not be diagnostic.

- Endoscopic drainage is the treatment of choice for sinonasal mucocoeles and carries low morbidity.

Acknowledgments. SEA thanks MM for guidance and advice in the writing process.

Author contributions. SEA: senior author, investigated the topic, collated the information and images, and produced the first draft. MM: co-author, assisted with selection of images and final editing of manuscript.

Funding. None.

Conflicts of interest. None.

1. Jang YJ, Kim SC. Pneumatization of the sphenoid sinus in children evaluated by magnetic resonance imaging. Am J Rhinology 2000;14(3):181-185. https://doi. org/10.2500/105065800782102771

2. Kösling S, Hintner M, Brandt S, et al. Mucoceles of the sphenoid sinus. Eur J Radiol 2004;51(1):1-5. https://doi.org/10.1016/j.ejrad.2003.09.002
3. Lund VJ, Harvey W, Meghji S, Harris M. Prostaglandin synthesis in the pathogenesis of fronto-ethmoidal mucoceles. Acta Oto-laryngol 1988;106(1-2):145-151. https://doi.org/10.3109/00016488809107382

4. Levy J, Monos T, Puterman M. Bilateral consecutive blindness due to sphenoid sinus mucocele with unilateral partial recovery. Can J Ophthalmol 2005;40(4):506-508.

5. Sundar U, Sharma AL, Yeolekar ME, Pahuja V. Sphenoidal sinus mucocoele presenting as mono-ocular painless loss of vision. Postgrad Med J 2004;80(939):40.

6. Yong W-W, Zhou S-H, Bao Y-Y. Sphenoid sinus mucocele presenting with oculomotor nerve palsy and affecting the functions of trigeminal nerve: A case report. Int J Clin Experimental Med 2015;8(9):16854-16857.

7. Haloi AK, Ditchfield M, Maixner W. Mucocele of the sphenoid sinus. Pediatric Radiol 2006;36(9):987-990. https://doi.org/10.1007/s00247-006-0243-x

8. Malard O, Gayet-Delacroix M, Jegoux F, Faure A, Bordure P, de Montreuil CB. Spontaneous sphenoid sinus mucocele revealed by meningitis and brain abscess in a 12-year-old child. Am J Neuroradiol 2004;25(5):873-875.

9. Lawson W, Reino AJ. Isolated sphenoid sinus disease: An analysis of 132 cases. Laryngoscope 1997;107(12):1590-1595. https://doi.org/10.1097/00005537199712000-00003

Accepted 1 February 2018. 